\title{
Does it matter where to run? intention to participate in destination marathon
}

\begin{abstract}
Purpose: The purpose of this study is to examine the effect of marathon enthusiasts' perceptions towards venue quality, race competition, organisation and service quality on their intention to participate in a destination marathon in the emerging region's context. It also seeks to investigate the mediating effect of perceived value and the moderating effect of intention to visit the destination on the intention to participate. Design/methodology/approach: Using purposive sampling technique, 177 valid Singapore marathon enthusiasts were sampled to look into their intention towards participating in destination marathon in Sarawak (marathon held in Kuching). The data were analysed using the partial least squares-structural equation modelling (PLSSEM). Findings: The results show that amongst the other determinants, perceived organisation and perceived service quality do not contribute to perceived value and intention to participate in destination marathon. Perceived value is found to mediate all path relationships except the relationship between perceived organisation and intention to participate. Moreover, the relationship between perceived value and intention to participate is significantly moderated by intention to tour Sarawak. Originality/value: This study makes a substantial contribution to the extant literature pertaining to destination tourism and value-based marketing in an emerging market. In particular, it highlights the importance of perceived value and the relevance of destination tourism in joining a sport event on foreign soil. The use of PLS-SEM also allows a rigorous assessment of the relationships under investigation and provides better estimations of the phenomenon.
\end{abstract}

Keyword: Destination marathon; Perceived value; Intention to tour; Self-determination theory; Tourism; PLS-SEM 